\title{
Influencia de la comunicación no verbal en las relaciones interpersonales
}

\author{
Maribel Sofía Pizarro Mostacero \\ mpizarro@unitru.edu.pe \\ Universidad Nacional de Trujillo - Perú \\ Dulio Oseda Gago \\ dosedag@unmsm.edu.pe \\ https://orcid.org/0000-0002-3136-6094 \\ Universidad Nacional Mayor de San Marcos - Perú
}

\section{RESUMEN}

La presente investigación tuvo como objetivo determinar la correlación entre la comunicación no verbal y las relaciones interpersonales en los estudiantes de lengua y literatura de la UNT 2020. La investigación es de tipo no experimental, transversal correlacional causal. Se aplicó un cuestionario de comunicación no verbal y relaciones interpersonales a una muestra censal de 212 estudiantes de la escuela de Lengua y Literatura. En los resultados se observó que el 96,2\% de estudiantes tienen un nivel bueno de comunicación no verbal, mientras que en relaciones interpersonales un 50,5\% se ubicó en nivel bueno, existiendo un $2,4 \%$ con nivel medio. Así mismo, se obtuvo un valor de "rho" = 0,392 y un valor de significancia de 0,000 menor que el nivel de confiabilidad de 0,05. Concluyéndose que existe correlación entre ambas variables. Por tanto, a mayor nivel de comunicación no verbal mayor nivel de relaciones interpersonales en los estudiantes de lengua y literatura de la UNT 2020.

Palabras clave: comunicación; comunicación no verbal; relaciones interpersonales. 


\title{
Influence of non-verbal communication in interpersonal relationships
}

\begin{abstract}
The objective of this research was to determine the correlation between non-verbal communication and interpersonal relationships in language and literature students at UNT 2020. The research is non-experimental, cross-sectional causal correlation. A non-verbal communication and interpersonal relationships questionnaire were applied to a census sample of 212 students from the School of Language and Literature. In the results, it was observed that $96.2 \%$ of students have a good level of non-verbal communication, while in interpersonal relationships $50.5 \%$ were at a good level, with $2.4 \%$ having a medium level. Likewise, a value of "rho" $=0.392$ and a significance value of 0.000 less than the reliability level of 0.05 was obtained. Concluding that there is a correlation between both variables. Therefore, the higher the level of non-verbal communication, the higher the level of interpersonal relationships in language and literature students at UNT 2020.
\end{abstract}

Keywords: communication; non-verbal communication; interpersonal relationships.

Artículo recibido: 10. Mayo. 2021 Aceptado para publicación: 28. junio. 2021 Correspondencia: mpizarro@unitru.edu.pe Conflictos de Interés: Ninguna que declarar 


\section{INTRODUCCIÓN}

A nivel mundial, se le da mayor importancia al lenguaje no verbal en un 55\%, un $38 \%$ a la vocalización, voz y dicción; y un 7\% a la palabra. El ser humano como especie se vale de la comunicación para poder interrelacionarse con los otros, siendo desde el principio el lenguaje no verbal el fundamento de la comunicación antes de existir el habla, los movimientos corporales, los gestos, los sonidos, han desempeñado el papel de establecer el contacto con los otros y dirigir indirectamente la relación con su semejante. Con la aparición del habla se reforzó la comunicación y se mantuvo la relación directa, pero el juego de palabras no termina de expresar para el otro si el contenido en el lenguaje oral es cierto o no, es por eso, que la interrelación humana basa el tipo de relaciones en base a lo que percibimos intuitivamente del lenguaje no verbal de ese inconstante que refleja realmente la intención del interlocutor, lo cual facilita el contacto con el otro. Pudiendo concluir que en la demostración de la comunicación entre las personas ocurre en gran medida por los componentes no verbales en específico: señales verbales y visuales, los cuales son procesados de manera inconsciente, siendo el asidero real de la aceptación o rechazo del otro.

En nuestra localidad, la Libertad es la región donde se observa mayores problemas de conflictos sociales, por ende, todas las instituciones deben de priorizar temas referentes a mejorar las relaciones personales, sociales e interacciones humanas, la falta de comunicación y forma de dirigirse a nuestros interlocutores son necesarios tener los conocimientos y la guía de saber dirigirnos a los otros; la naturaleza de una relación depende de una eficiente comunicación verbal y no verbal.

Según mi experiencia del día a día en las aulas de la Escuela de Lengua y Literatura de la UNT, con los estudiantes, escuchaba decir: Me falta expresión y soltura corporal, mis compañeros hacen gestos inapropiados durante la interacción comunicativa, suelen no mantenerse firmes al momento de expresarse, Me avergüenzo de que me miren a los ojos, siento temblor en mis manos, siento frío al exponer, mi cara se pone roja, tiembla mi voz, Mi voz sale intermitente y baja, etc. Situaciones que motivaron la presente investigación frente a los problemas de comunicación no verbal y las consecuencias generadas por tales actos involuntarios y voluntarios, la existencia de malentendidos, las deficientes relaciones interpersonales entre estudiantes.

La asociación de estas dos variables de estudio tiene por finalidad proponer mejoras en la relación humana específicamente en el ámbito educativo de nivel superior por estudiantes 
de Pregrado de la Escuela de Lengua y Literatura, que son el eje fundamental para la mejora social, pues es transcendental en los futuros docentes. Un buen pedagogo debe saber comunicarse efectivamente con sus estudiantes, de no ser así, a pesar de ser muy competente en su rubro, no llegará a cumplir el objetivo de la enseñanza-aprendizaje de una manera eficiente y eficaz.

Finalmente, todo tema que involucre o que tenga como eje al ser humano, es de gran importancia estudiarlo, ya que, a partir del estudio, se conocerá muchos aspectos del cual aún se siguen desconociendo.

Ahora se formula el problema general de la tesis que literalmente es el siguiente: ¿Existe influencia entre la comunicación no verbal y las relaciones interpersonales en los estudiantes de la Escuela de Educación Secundaria, Especialidad Lengua y Literatura de la Universidad Nacional de Trujillo en el año 2020? Problemas específicos: ¿Existe influencia de la comunicación no verbal en la dimensión habilidades sociales? ¿Existe influencia de la comunicación no verbal en la dimensión Estilos de liderazgo?

En cuanto a la justificación, según (Hernández, Fernández, \& Baptista, 2010) se tiene los siguientes criterios: Conveniencia, la investigación en toda su amplitud fue conveniente por tratarse de situaciones vivenciales en las que se desenvuelve todo ser humano en su proceso de humanización.

Relevancia social, la investigación se abordó con un grupo de jóvenes de la Universidad Nacional de Trujillo de la escuela de lengua y literatura; ellos se beneficiaron por ser un tema del acontecer diario de los cuales tenemos distintos comportamientos visibles. Valor teórico, la investigación sirvió para conocer qué tan importantes son ciertas acciones del ser humano en la interacción social. Valor práctico, la investigación ayudó a la comunidad universitaria a reconocer una amplia variedad de emociones del ser humano en su contexto social. Utilidad metodológica, la investigación en todo su contenido se guio por el método científico.

El objetivo general: Determinar la influencia entre la comunicación no verbal y las relaciones interpersonales en los estudiantes de la Escuela de Educación Secundaria, Especialidad Lengua y Literatura de la Universidad Nacional de Trujillo en el año 2020. Y los objetivos específicos: Determinar la influencia de la comunicación no verbal en la dimensión habilidades sociales. Determinar la influencia de la comunicación no verbal en la dimensión estilos de liderazgo Y la hipótesis general: Existe influencia entre la 
comunicación no verbal y las relaciones interpersonales en los estudiantes de la Escuela de Educación Secundaria, Especialidad Lengua y Literatura de la Universidad Nacional de Trujillo en el año 2020. Hipótesis específicas: Existe influencia de la comunicación no verbal en la dimensión habilidades sociales. Existe influencia de la comunicación no verbal en la dimensión estilos de liderazgo.

Respecto a las bases teórico científicas, el lenguaje corporal referente a la forma de mirar, al cómo manifestar nuestros gestos, el ritmo de voz, su tonalidad, la posición del cuerpo expresamos muchas emociones con el que nos comunicamos en forma constante. Los conjuntos de estos elementos reciben el nombre de comunicación no verbal. (Rulicki \& Cherny, 2007). La cual es la variable 1 de la presente investigación.

Según (Baró T. , 2012). Cuando se refiere al lenguaje no verbal, la inclusión de movimientos, gesticulaciones que manifiesta todo ser humano en diferentes situaciones comunicativas, el individuo utiliza el lenguaje corporal, que muchas veces se considera sinónimo de comunicación no verbal, pero ésta tiene otra dimensión más amplia que abarca la conducta táctil, el entorno, los espacios y contex to geográfico tan imprescindible para el hombre.

En cuanto a la variable Relaciones interpersonales (Pereira, Trentino, Alves, \& Puggina, 2019) Las relaciones interpersonales con los compañeros de clase son vitales e importantes. Autores identificaron que los estudiantes que describieron positivamente la relación con los compañeros de clase referidos para percibir cooperación, amistad y lazos que superan las instituciones, Sin embargo, hubo quienes evaluaron negativamente La relación con los compañeros de clase, debido a diferencias en los valores. y estilos de vida, siendo estos desacuerdos factores fundamentales por evasión. [trad].

Para Silveira, (2014) citado por (Vásquez, 2018, p. 15) Se define a las relaciones interpersonales como una interacción eficaz entre dos o más personas que fomentan un medio favorable para la comunicación. Este aspecto es considerado el inicio de todo aprendizaje las cuales suelen generar molestias o incomodidad en los seres, a la vez permiten un crecimiento en el hombre ya sea en sus relaciones interpersonales, amicales, familiares y de contexto frente a diferentes comportamientos, cambios de emociones, opiniones del ser humano.

La importancia de las relaciones interpersonales radica en que contribuyen al desarrollo cognitivo y social entre los seres humanos. Lo mejor que puede pasarle al hombre es la 
relación con otras personas. Por ello, debido a las grandes limitaciones observadas en los estudiantes universitarios sería necesario sensibilizar al docente y estudiantes para promover una correcta relación.

Las principales teorías que fundamentan las relaciones interpersonales son la teoría de Carl Rogers y la teoría de las inteligencias múltiples de Howard Gardner, donde se incluye la inteligencia interpersonal, como parte de esta teoría.

Conociendo la importancia de la comunicación en las relaciones interpersonales, necesitamos tener en cuenta las habilidades sociales, determinando como dimensiones: asertividad, empatía, escucha activa y reconocimiento de sus errores y aciertos.

Por último, proponer un programa de talleres de estrategias de comunicación no verbal para mejorar las relaciones interpersonales a nivel remoto.

\section{ESTRATEGIAS METODOLÓGICAS O MATERIALES Y MÉTODOS}

La investigación pertenece al enfoque cuantitativo, tipo aplicada y de nivel correlacional, la investigación hizo uso del método general denominado método científico y de los métodos específicos como es el descriptivo, y el hipotético deductivo. El diseño de la presente investigación fue correlacional causal o predictivo, este diseño según Hernández, Fernández y Baptista (2010). Describe relaciones entre dos o más categorías, conceptos o variables en un momento determinado. A veces, únicamente en términos correlacionales, otras en función de la relación causa-efecto (causales).

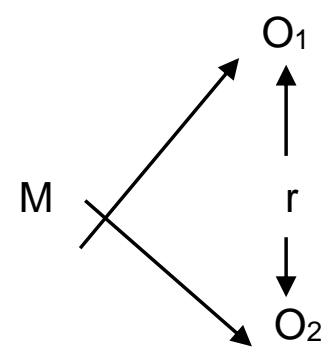

\section{Donde:}

$\mathbf{M}=$ Muestra conformada por los estudiantes de la especialidad de Lengua y Literatura de la Universidad Nacional de Trujillo en el año 2020.

$\mathbf{O}_{1}=$ Observación de la variable independiente: Comunicación no verbal

$\mathbf{O}_{2}=$ Observación de la variable dependiente: Relaciones interpersonales.

$\mathbf{r}=$ Correlación entre las variables, Comunicación no verbal y relaciones interpersonales. 
La población objeto de estudio del presente trabajo estuvo comprendido por 212 estudiantes de la especialidad de Lengua y Literatura de la Universidad Nacional de Trujillo 2020. La muestra de estudio es una muestra censal que constará de 212 estudiantes de la especialidad de Lengua y Literatura de la Universidad Nacional de Trujillo como única Universidad Estatal de la ciudad de Trujillo que brinda dicha especialidad. Se seleccionará el $100 \%$ de la población al considerarla una cantidad manejable de estudiantes. En tal sentido (Ramirez, 1997) menciona que la muestra censal es aquella en las cuales todas las unidades de investigación son seleccionadas como muestra (p.77). De tal forma la población a estudiar se denominará censal por ser universo, población y muestra. En vista que la población no es numerosa (López, 1998), opina que "la muestra censal es aquella porción que representa toda la población" (p.123).

\section{RESULTADOS Y DISCUSIÓN}

A continuación, se presenta los resultados de la variable 1 Comunicación no verbal aplicado a los 212 estudiantes de la especialidad de Lengua y Literatura de la Universidad Nacional de Trujillo 2020. Los cuales se visualizan en la siguiente tabla.

Tabla 1: Nivel alcanzado en comunicación no verbal en los estudiantes de la Universidad Nacional de Trujillo

\begin{tabular}{llcc}
\hline Nivel & $\mathbf{N}^{\circ}$ & $\%$ \\
\hline Bajo & 0 & 0.0 \\
\hline Medio & 8 & 3.8 \\
\hline Bueno & 204 & 96.2 \\
\hline Excelente & 0 & 0.0 \\
\hline Total & 212 & 100.0 \\
\hline
\end{tabular}

\section{Interpretación:}

Fuente. Datos obtenidos de la aplicación del test

De la tabla 1, se puede apreciar que la mayoría de los estudiantes, 96.2\% (204 estudiantes) se encuentran en un nivel bueno de comunicación no verbal y sólo el 3.8\% (8 estudiantes) se encuentra con nivel medio. Estos resultados difieren a la investigación realizada por (Galván, 2018) con estudiantes de la especialidad de Agropecuarias de la Universidad Enrique Guzmán y Valle que obtuvieron un nivel medio de comunicación asertiva equivalente al 50\%. El cual como menciona (Álvarez, 2012), considera que la comunicación no verbal en el ambiente educativo es básica. Por ello, la comunicación humana que se ocupa de las relaciones interpersonales es trascendental para fomentar una 
comunicación de calidad que conlleven a una mejor forma de vida en todos los aspectos y en especial en el ámbito universitario. Para (Davis, 1998), menciona que la comunicación no verbal es esencial y crucial en todo individuo por sus manifestaciones de conducta y expresiones en el acto comunicativo. Así mismo (Baró, 2012) refiere que el lenguaje no verbal tanto en la inclusión de movimientos, gesticulaciones que manifiesta todo ser en diferentes actos comunicativos tiene una dimensión amplia abarcando diferentes conductas que son importantes en toda interacción.

Tabla 2: Nivel alcanzado en relaciones interpersonales en los estudiantes de la Universidad Nacional de Trujillo

\begin{tabular}{llc|c}
\hline Nivel & $\mathbf{N}^{\circ}$ & $\%$ \\
\hline Bajo & 0 & 0.0 \\
\hline Medio & 5 & 2.4 \\
\hline Bueno & 107 & 50.5 \\
\hline Excelente & 100 & 47.2 \\
\hline Total & 212 & 100.0 \\
\hline
\end{tabular}

Fuente. Datos obtenidos de la aplicación del test

\section{Interpretación:}

De la tabla 2, se puede apreciar que la mayoría, el 50.5\% (107 estudiantes) tienen un nivel bueno en relaciones interpersonales; seguido del 47.2\% (100 estudiantes) con un nivel excelente. Resultados favorables que, según Pereira, et al (2019), las relaciones interpersonales entre estudiantes son fundamentales, según sus investigaciones manifiestan que los estudiantes logran lazos fuertes de amistad y compañerismo. Pero también otros investigadores manifiestan resultados desfavorables argumentando diferencias en valores y estilos de vida. Para Silveira, (2014) citado por (Vásquez, 2018, p. 15) "Refiere a las relaciones interpersonales como una interacción eficaz entre dos o más personas que fomentan un medio favorable para la comunicación." Por ello, consideramos un aprendizaje continuo en el ser humano por los diferentes comportamientos, estados de ánimo, puntos de vista en el proceso comunicativo que conlleva a las relaciones interpersonales. 
Tabla 3: Relación de la comunicación no verbal y las relaciones interpersonales en los estudiantes de la Universidad Nacional de Trujillo

\begin{tabular}{lllcc}
\hline & & & $\begin{array}{c}\text { Comunicación } \\
\text { no verbal }\end{array}$ & $\begin{array}{c}\text { Relaciones } \\
\text { interpersonales }\end{array}$ \\
\hline $\begin{array}{l}\text { Rho de } \\
\text { Spearman }\end{array}$ & $\begin{array}{l}\text { Comunicación No } \\
\text { verbal }\end{array}$ & $\begin{array}{l}\text { Coeficiente de } \\
\text { correlación }\end{array}$ & 1,000 &, $392^{* *}$ \\
\cline { 2 - 5 } & & Sig. (bilateral) & $\cdot$ &, 000 \\
\cline { 2 - 5 } & $\mathrm{N}$ & 212 & 212 \\
\cline { 2 - 5 } & $\begin{array}{l}\text { Relaciones } \\
\text { interpersonales }\end{array}$ & $\begin{array}{l}\text { Coeficiente de } \\
\text { correlación }\end{array}$ &, $392^{* *}$ & 1,000 \\
\cline { 2 - 5 } & Sig. (bilateral) &, 000 & \\
\cline { 2 - 5 } & $\mathrm{N}$ & 212 & 212 \\
\hline
\end{tabular}

**. La correlación es significativa en el nivel 0,01 (bilateral).

Fuente. Datos obtenidos de la aplicación del test

De la tabla 3, se puede observar que, si existe relación significativa entre la comunicación no verbal y las relaciones interpersonales, encontrándose en la prueba de correlación de Spearman, un valor de 0.392 con una significancia de $0.000(\mathrm{p}<0.05)$.

Tabla 4: Influencia de la comunicación no verbal en las relaciones interpersonales en los estudiantes de la Universidad Nacional de Trujillo

\begin{tabular}{llccc|c|c}
\hline \multicolumn{7}{c}{ ANOVA $^{\text {a }}$} \\
\hline \multirow{2}{*}{ Modelo } & $\begin{array}{c}\text { Suma de } \\
\text { cuadrados }\end{array}$ & gl & $\begin{array}{c}\text { Media } \\
\text { cuadrática }\end{array}$ & F & Sig. \\
\hline 1 & Regresión & 4662,346 & 1 & 4662,346 & 43,869 &, $000^{\mathrm{b}}$ \\
\cline { 2 - 7 } & Residuo & 22318,386 & 210 & 106,278 & & \\
\cline { 2 - 8 } & Total & 26980,731 & 211 & & & \\
\hline
\end{tabular}

a. Variable dependiente: Relaciones interpersonales

b. Predictores: (Constante), Comunicación no verbal

\begin{tabular}{|c|c|c|c|c|c|c|}
\hline \multicolumn{7}{|c|}{ Coeficientes $^{\mathbf{a}}$} \\
\hline & \multirow{2}{*}{ Modelo } & \multicolumn{2}{|c|}{$\begin{array}{l}\text { Coeficientes no } \\
\text { estandarizados }\end{array}$} & \multirow{2}{*}{$\begin{array}{c}\begin{array}{c}\text { Coeficientes } \\
\text { estandarizados }\end{array} \\
\text { Beta }\end{array}$} & \multirow{2}{*}{$\mathbf{t}$} & \multirow{2}{*}{ Sig. } \\
\hline & & B & $\begin{array}{l}\text { Desv. } \\
\text { Error }\end{array}$ & & & \\
\hline \multirow[t]{2}{*}{1} & (Constante) & 43,418 & 10,114 & & 4,293 &, 000 \\
\hline & $\begin{array}{l}\text { Comunicación no } \\
\text { verbal }\end{array}$ & ,778 & ,117 & ,416 & 6,623 &, 000 \\
\hline
\end{tabular}

a. Variable dependiente: Relaciones interpersonales

Existe evidencia a un nivel del $95 \%$ de confianza, que la comunicación no verbal influye positivamente en las relaciones interpersonales $(\mathrm{Sig}<0.05)$ 


\begin{tabular}{lc|c|c|c|c}
\hline \multicolumn{6}{c}{ Resumen del modelo $^{\mathbf{b}}$} \\
\hline Modelo & $\mathbf{R}$ & $\begin{array}{c}\mathbf{R} \\
\text { cuadrado }\end{array}$ & $\begin{array}{c}\text { R cuadrado } \\
\text { ajustado }\end{array}$ & $\begin{array}{c}\text { Error } \\
\text { estándar de } \\
\text { la estimación }\end{array}$ & $\begin{array}{c}\text { Durbin- } \\
\text { Watson }\end{array}$ \\
\hline 1 &, $416^{\mathrm{a}}$ &, 173 &, 169 & 10,30912 & 1,753 \\
\hline
\end{tabular}

a. Predictores: (Constante), Comunicación no verbal

b. Variable dependiente: Relaciones interpersonales

De la tabla se verifica que la comunicación no verbal influye en un $17.3 \%$ en las relaciones interpersonal, siendo $82.7 \%$ influenciado por otros factores

Figura 1: Dispersión de los puntajes de la Comunicación no verbal y las Relaciones Interpersonales

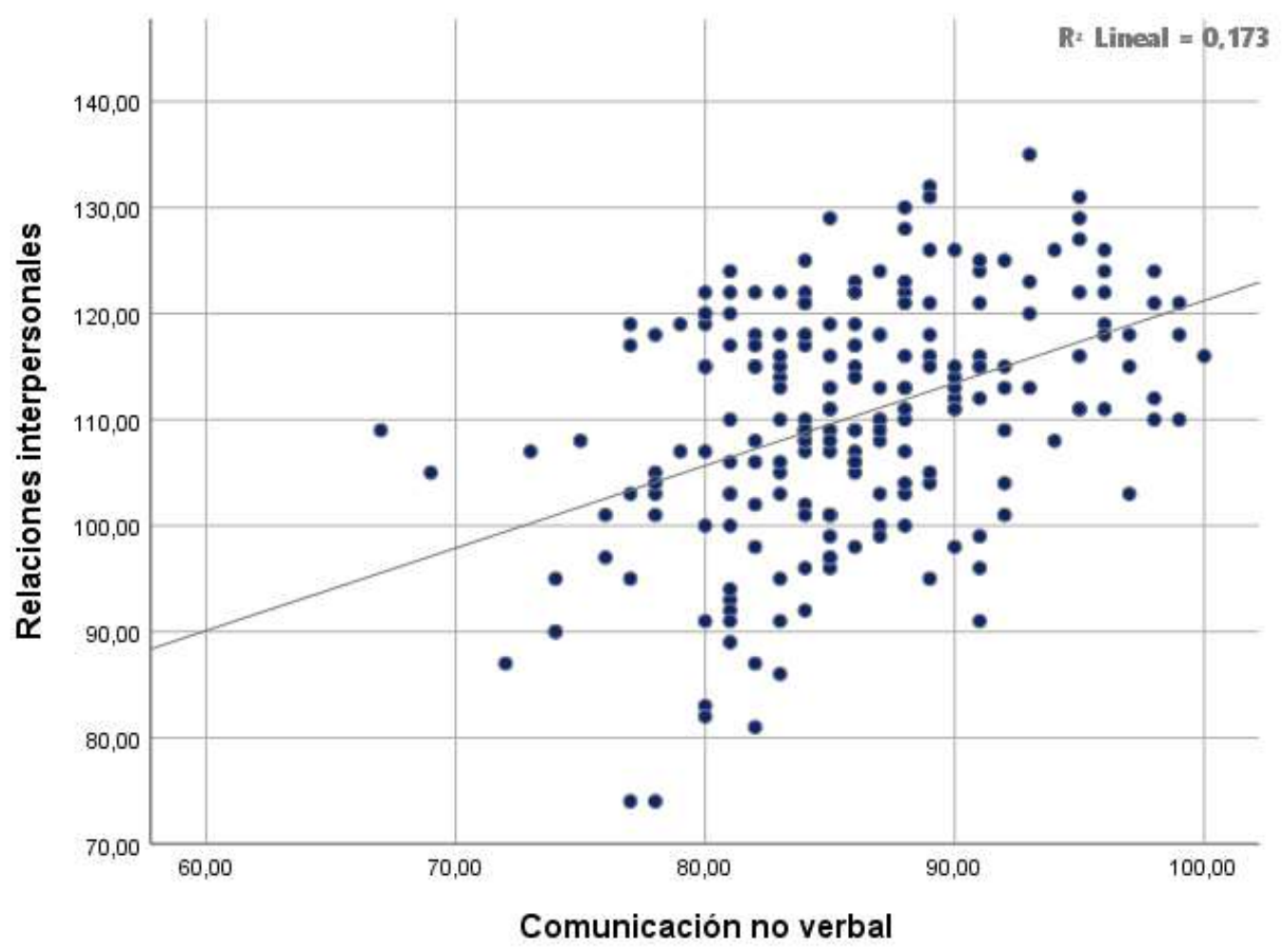

Fuente. Datos obtenidos de la aplicación del test

De la figura 1, se puede observar que, si existe relación positiva, entre la Comunicación no verbal y las Relaciones Interpersonales, indicando que, a mejor desarrollo de la Comunicación no verbal, mejor serán las Relaciones Interpersonales. Verificándose que la comunicación no verbal influye $17.3 \%$ en las relaciones interpersonales. 


\section{DISCUSIÓN}

En relación con los resultados obtenidos se puede observar que, si existe relación significativa entre la comunicación no verbal y las relaciones interpersonales, encontrándose en la prueba de correlación de Spearman, un valor de 0.392 con una significancia de $0.000(\mathrm{p}<0.05)$. Frente a lo mencionado se acepta la hipótesis de investigación, donde refiere que existe influencia entre la comunicación no verbal y las relaciones interpersonales en los estudiantes de la Escuela de Educación Secundaria, Especialidad Lengua y Literatura de la Universidad Nacional de Trujillo en el año 2020. Asu vez Cano, L (2018) menciona en su tesis de posgrado Comunicación asertiva y relaciones interpersonales en docentes de la Red 6, UGEL 05. San Juan de Lurigancho2018, que obtuvo como resultado una relación significativa entre la comunicación asertiva y relaciones interpersonales en docentes de nivel primaria de la Red 6, UGEL 05, San Juan de Lurigancho. 2018. siendo que el coeficiente de correlación Tau_b de Kendall de 0.772 , demostrando una alta asociación entre las variables.

De igual manera Aguilar, M (2018) en su investigación Comunicación asertiva y relaciones interpersonales en docentes de secundaria de la institución educativa de los Olivos, 2018; sus resultados determinaron que la comunicación asertiva se relaciona (Rho $=0$, 753) y significativamente $(\mathrm{p}=0.000)$ con las relaciones interpersonales en docentes de instituciones educativas de Los Olivos, 2018. Dichos resultados son similares a las investigaciones anteriores con la diferencia que la investigación fue a docentes de Educación Básica. Significa entonces que los procesos interpersonales al interior de las Instituciones educativas ya sea de Educación Básica o Educación Superior Universitaria son muy importantes para el estudio de la comunicación no verbal, pues en todo proceso comunicativo según el estudio realizado por el antropólogo Albert Mehrabian quien enunció una regla sobre la comunicación conocida como la 7-38-55. 7\% palabras, 38\% lenguaje paraverbal y 55\% lenguaje corporal, exclusivamente en conversaciones con alto contenido emocional.

Estos resultados son corroborados por Galván, V. (2018) en su tesis doctoral Relaciones Interpersonales y el nivel de asertividad en los alumnos del VII ciclo de la Universidad Nacional de Educación Enrique Guzmán y Valle, concluye que las habilidades interpersonales tienen una relación directa con el nivel de asertividad en los alumnos del VII ciclo de la Especialidad de Agropecuaria de la Universidad Nacional de Educación 
Enrique Guzmán y Valle. Nos hace reflexionar que se enriquece las relaciones interpersonales poniendo en práctica una comunicación asertiva que todo ser humano y grupo humano desea especialmente en los estudiantes de nivel superior.

Asu vez la relación alumno-alumno como afirma (Vuelvas,1997); en la etapa de adolescentes el joven necesita conformar su identidad a partir de un proceso de individualización, es él y solo él, tratando de ser único con otras personas, buscando diferencias de los demás. Todos estos momentos son importantes especialmente la comunicación no verbal que representa la parte fundamental en toda interacción humana, en las diferentes etapas del desarrollo humano.

Estos resultados son fortalecidos con la teoría de (Bambacero \& Shokrpour, 2017); la comunicación no verbal es altamente confiable en el proceso de comunicación porque las señas no verbales revelan la interacción del emisor en el proceso comunicativo reflejando sus reacciones emocionales. Con base a estas afirmaciones de estudio recomiendan la atención a las habilidades de comunicación no verbal, pues determina un cambio favorable en las buenas relaciones interpersonales de todo ser humano.

En cuanto a variable Relaciones interpersonales (Pereira, Trentino, Alves, \& Puggina, 2019) Las relaciones interpersonales con los compañeros de clase son vitales e importantes. Autores identificaron que los estudiantes que describieron positivamente la relación con los compañeros de clase referidos para percibir cooperación, amistad y lazos que superan las instituciones, Sin embargo, hubo quienes evaluaron negativamente La relación con los compañeros de clase, debido a diferencias en los valores. y estilos de vida, siendo estos desacuerdos factores fundamentales por evasión. [trad].

Para Silveira, (2014) citado por (Vásquez, 2018, p. 15) Se define a las relaciones interpersonales como una interacción eficaz entre dos o más personas que fomentan un medio favorable para la comunicación. Este aspecto es considerado el inicio de todo aprendizaje las cuales suelen generar molestias o incomodidad en los seres, a la vez permiten un crecimiento en el hombre ya sea en sus relaciones interpersonales, amicales, familiares y de contexto frente a diferentes comportamientos, cambios de emociones, opiniones del ser humano.

Para (Pedroza, 2012) considera que el contexto social con toda su diversidad para que podamos hacer relaciones interpersonales de manera democrática se debe aceptar al otro 
en su diferencia y lo primero debemos ser conscientes de nuestras posibilidades como educadores.

\section{CONCLUSIÓN O CONSIDERACIONES FINALES}

Existe influencia directa y significativa la comunicación no verbal y las relaciones interpersonales, con un coeficiente de correlación de 0.392 y un coeficiente R2 (0.173). observándose que, a mejor desarrollo de la Paralingüística, mejor será el desarrollo del Estilo de Liderazgo.

En las dimensiones de comunicación no verbal se puede evidenciar que en Kinésica y paralingüística, los estudiantes presentan buen nivel encontrándose respectivamente $88.7 \%$ (188 estudiantes) y $82.1 \%$ (174 estudiantes); sin embargo, en la proxémica aún encontramos al $44.8 \%$ (95 estudiantes) en nivel medio.

En las relaciones interpersonales tenemos a la mitad con nivel bueno y el 47.2\% (100 estudiantes) con un nivel excelente, existiendo sólo un 2.4\% (5 estudiantes) con nivel medio.

En las dimensiones de relaciones interpersonales, se evidencia que, en habilidades sociales el $56.1 \%$ presenta excelente desarrollo y el $41.5 \%$ buen desarrollo, para la dimensión estilos de liderazgo el $62.3 \%$ presenta nivel bueno y sólo el $30.2 \%$ se ubica en nivel excelente, siendo un grupo a quienes hay que reforzar.

Existe influencia directa y significativa baja de la Comunicación no verbal en la dimensión habilidades sociales con un coeficiente de correlación de 0.388 y un coeficiente R2 (0.165). Indicando que, a mejor desarrollo de la comunicación no verbal, mejor será el desarrollo de habilidades sociales.

Existe influencia directa y significativa baja de la comunicación no verbal y la dimensión estilos de liderazgo, con un coeficiente de correlación de 0.341 y un coeficiente R2 (0.129). Indicando que, la comunicación no verbal influye en $12.9 \%$ en la variabilidad de los estilos de liderazgo.

\section{LISTA DE REFERENCIAS}

Aguilar, M. (2018). Comunicación asertiva y relaciones interpersonales en docentes de secundaria de la Institución educativa de los Olivos. Universidad César Vallejo, Lima

Baró, T. (2012). La gran guía del lenguaje no verbal. Barcelona: Paidós 
Bambacero, F., \& Shokrpour, N. (2017). The impact of the teachers' non-verbal communication on success in. The impact of the teachers' non-verbal communication on success in, 5(2), 51-59

Cano, L. (2018). Comunicación asertiva y relaciones interpersonales en docentes de la Red 6, UGEL 05. San Juan de Lurigancho-2018. Lima: Universidad César Vallejo.

Davis, F. (1976). La comunicación no verbal. Madrid: Alianza editorial.

Galván, V. (2018). Relaciones interpersonales y el nivel de asertividad en los alumnos del VII ciclo de la especialidad de agropecuaria de la Universidad Nacional de Educación Enrique Guzmán y Valle. Lima.

Hernández, R., Fernández, C., \& Baptista, P. (2010). Metodología de la investigación. México: $5^{\circ}$ ed. Mc Graw Hill

Javier, A., Santa María, H., Norabuena, R., \& Jara, N. (2019). Acción tutorial para el desarrollo de las habilidades sociales en estudiantes universitarios. 7(1), 185-200. doi:http://dx.doi.org/10.20511/pyr2019.v7n1.261

López, J. (1998). Procesos de investigación. Caracas, Venezuela: Panapo.

Rulicki, S., \& Cherny, M. (2007). Comunicación no verbal. Buenos Aires:

Pereira, T., Trentino, J., Alves, F., \& Puggina, A. (2019). Factors that affecting nursing students quality of interpersonal relationships. $E A N, 23(1)$. http://dx doi:10.1590/2177-9465-EAN-2018-0159

Pedroza, L. S. (2012). Relações Interpessoais: (4ta ed.). Cuiabá - MT, Brasil: Universidade Federal de Mato Grosso/Rede e-Tec Brasil.

Ramirez, T. (1997). Cómo hacer un proyecto de investigación. Caracas, Venezuela: Panapo

Vasquez, J. (2018). Relaciones interpersonales y motivación laboral en los docentes de la Institución Educativa $N^{\circ} 80382$ Carlos Alberto Olivares. Chepén-2017. Trujillo : Universidad César Vallejo.

Vuelvas, B. (1997). La juventud actual Comienzo o fin de una época”, Memorias del $2^{\circ}$ Congreso Nacional de Orientación Educativa. 